\title{
Analysis of Vertical Irregularities and Dynamic Forces on the Switch Frogs of the Underground Railway
}

\author{
Volodymyr Boiko*, Vitalii Molchanov, Volodymyr Tverdomed, and Olena Oliinyk \\ State University of Infrastructure and Technologies, 9 Kyrylivska St., 04071 Kyiv, Ukraine
}

\begin{abstract}
This article presents the analysis of vertical irregularities and dynamic forces on the switch frogs in operating conditions on the main tracks of Kiev underground railway. For main railways the deterioration problem of tongues, framed rails, switch frogs and as a consequence formation of the character in interaction on the railroad switches is quite widely studied by different scientists, but for conditions of railway underground such researches are practically absent. However, in order to use metal elements of switches rationally and efficiently, the forecast of their service operation and replacement planning, it is necessary to estimate the level of dynamic forces within the most deteriorated elements of switches at different operational stages. This problem is particularly relevant in conditions of financial restriction for track facilities. The studies allowed determining the form, parameters and basic patterns of the irregularities formation in the rolling zone on switch frogs P65 and P50 mark of crossing $1 / 9$ on wooden beams under different operating conditions, which is the basis for determining vertical dynamic forces of interaction within the switch frog subject to setting maximum permissible speeds of movement.
\end{abstract}

\section{Introduction}

Vertical irregularities on the rolling surface of switch frogs are one of the main disturbing factors in the formation of the oscillating process of the interaction between the wheels of the rolling stock and tracks within the most vulnerable zone of switches. This zone for ordinary switch frogs is located from the frog throat to the intersection of the triblet 20-40 $\mathrm{mm}$, where the wheel completely goes to the rolling on the triblet surface. In this zone, due to the rapid deterioration of narrow contact areas in the check rail and the triblet, irregularities are quickly formed during the operation causing the formation of additional inertial forces of wheel and rail interaction. In this case, the emerging forces of interaction have a value, exceeding similar forces within the normal track significantly.

During the diagnostic of switch frogs, the control of deterioration only in certain sections does not provide the objective assessment of power loading. According to this, there is necessary to know the trajectory of wheel rolling in vertical. This allows simulating

* Corresponding author: zkks@,ukr.net 
the nature of the interaction, determining the level of dynamic forces and setting the permissible speeds of railway vehicles.

Therefore, the analysis of vertical irregularities on switch frogs is a necessary condition for studying the issue of power dynamics on switches.

Many scholars have conducted this scientific problem having carried out a number of experimental and theoretical studies and they have proposed different approaches to the definition of vertical irregularities in the switching section.

Researches of prof. Yakovlev V. F. [1] define main characteristics of the irregularity shape in describing of the wheel trajectory: the form and parameters of irregularity (length, depth and slopes of irregularities).

Prof. Danilenko E.I. [2] has carried out large experimental researches about trajectories of wheel rolling on the switch frogs of different designs and stamps that were set for different conditions in sections of main and industrial transport. A number of average irregularities has been established for various structures and types of switch frogs on timbers $(1 / 5,1 / 6,1 / 7,1 / 9$ and 1/11) due to the different average deterioration and the deterioration of maximum.

There are well-known studies of switches on Ukrainian railways [3, 4, 5], where the formation of vertical irregularities on switch frogs and the estimation of power interaction of the "vehicle-track" system are considered.

Scientific works by Aniołek K., Herian J. [6] only consider a single cross section of the switch frog in the simulation of power interaction.

Zeng Z. and others [7] consider the wheel-rail contact parameters for undeteriorated switch frogs along the longitudinal direction of movement and deteriorated profiles of wheels.

J. Drozdziel and B. Sowinski [8] consider the irregularity formed on the base of the diameter geometry measured in the fixed sections of switch frogs.

In the scientific work of Jingmang $\mathrm{Xu}$ [9] the conditions of interaction within the arrow part of the switch with different deterioration characteristics have been modeled allowing to design the construction rationally.

Above-mentioned studies were mainly conducted for switches being operated on trunk transport, at the same time the conditions of switches operation in the underground railway have significant differences, and therefore require a separate research.

\section{Measurement of vertical irregularities on the switch frogs}

Investigations of vertical irregularities have been carried out in real conditions of the underground railway operation. In which case switches operated by the ground and underground zones of main underground railway tracks have been used.

Measurements have been carried out at 37 switches, including:

- 22 switches P50 mark of crossing 1/9;

- 15 switches P65 mark of crossing 1/9.

The selection of switches had been carried out according to the difference of characteristics in the tonnage missed during operation, and different operating conditions (the direction movement of trains - trailing or facing direction, different speeds, etc.).

The set speed of the movement $\mathrm{V}$ on switches is $V_{\max }=80 \mathrm{~km} / \mathrm{h}$ in the direction, $V_{\max }=$ $40 \mathrm{~km} / \mathrm{h}$ in the lateral direction. Maximum axial loadings were up to $P_{o}=14$ tons. The loading capacity $G$ million tons per year of the underground sections was 21-25million tons per year for switch frogs P50 and 17 million tons per year for P65. The capacity tonnage $T$ from the beginning to the time of investigations was from 13 to 133 million tons on the switch frogs P50, and from 25 to 106 million tons on P65. At which point switches with a 
preferred trailing movement direction of P50 and P65 there were investigated 13 and 10 correspondingly, and with facing movement direction 9 and 5.

Measurements of irregularities have only been performed in the direct direction of movement along the switching (main flow).

A mechanical device - a trajectory tracer - was used for measurements of rolling trajectories. The trajectory tracer consists of a semi-automatic device with a recording device that continuously records the position of the gravity center of the measuring wheel over the predominant direction of movement on the rolling surface along the switch frog.

To fix the characteristic points along the length of the switch frog, layout was made according to the scheme presented in Fig. 1

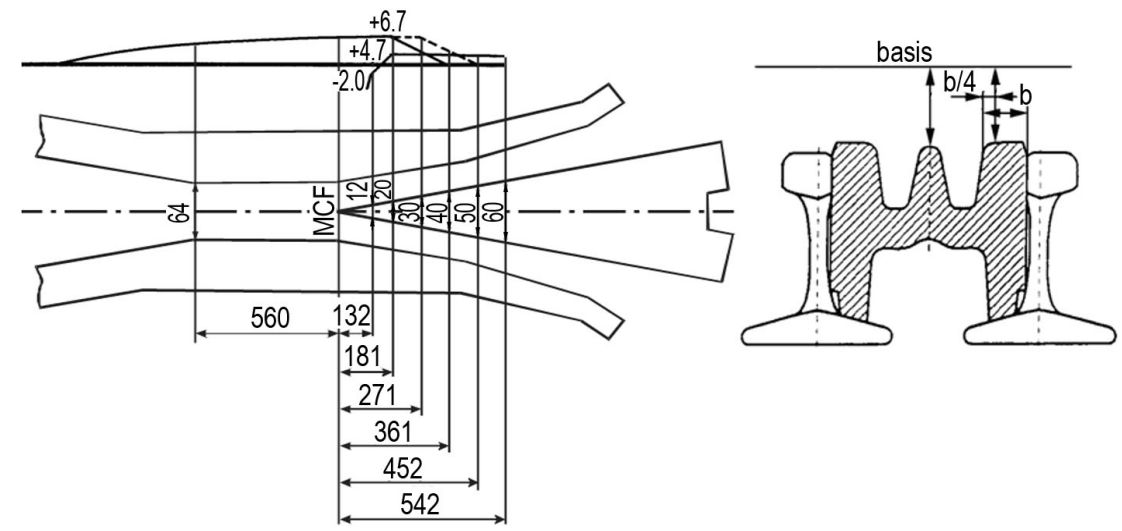

Fig. 1. The scheme of typical cross-sectional marking for measuring vertical irregularities of switch frogs, mark of crossings $1 / 9$

The vertical irregularity on the switch frog represents the trajectory of the center movement of the measuring wheel, which has a rolling surface according to the shape of the wheel rim. For measurements there was used a roller sharpened under the profile of a new wheel (it is possible to use the wheel profile with any condition of deterioration).

Results of operational studies about vertical irregularities on the rolling surface of the switch frogs have been represented in the form of natural trajectories of rolling from the intersection of the cast part of the check rail to the core section of $60 \mathrm{~mm}$ and defining of tonnage which was skipped according to the design in the measurement period.

For the analysis of trends in the development of irregularities at different stages of operation, all received vertical irregularities have been grouped depending on the type of switch frogs, the capacity of tonnage and the preferred direction of the train movement. For each group there have been determined the shape and parameters of irregularities depending on the tonnage at different stages of operation.

Mathematical processing of the statistical material was performed after systematizing of irregularities according to operational characteristics, and groups of irregularities have been obtained according to different stages of frog deterioration:

1) the initial period of operation (approximately after 1-1.5 years of operation);

2) the medium deterioration condition (approximately after 60-75 million tons of capacity tonnage);

3 ) the deterioration condition closing to the maximum (approximately after 100-120 million tons of capacity tonnage).

The average trajectory of rolling by the center of the wheel along the switch frog is shown in Fig. 2, 3.

Obtained vertical irregularities for different conditions and stages of operation became the basis for calculating forces of interaction within switch frogs. 
movement towards the connection

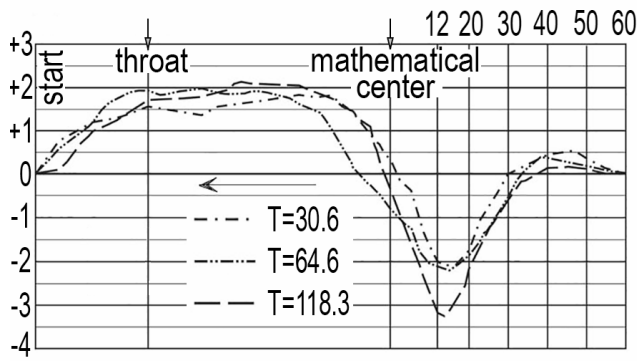

movement in the direction of the branching

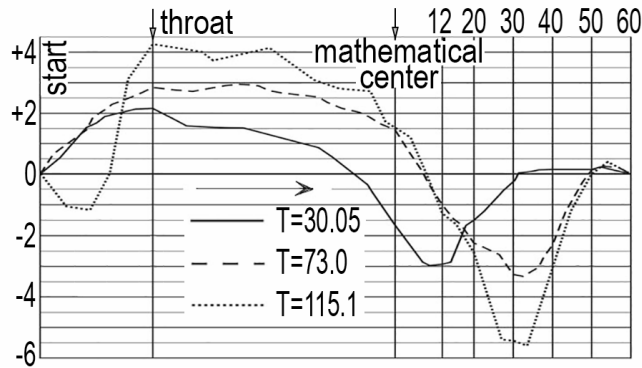

Fig. 2. Average vertical irregularities for the switch frogs P50, mark of crossings $1 / 9$ at different stages of operation.

movement towards the connection

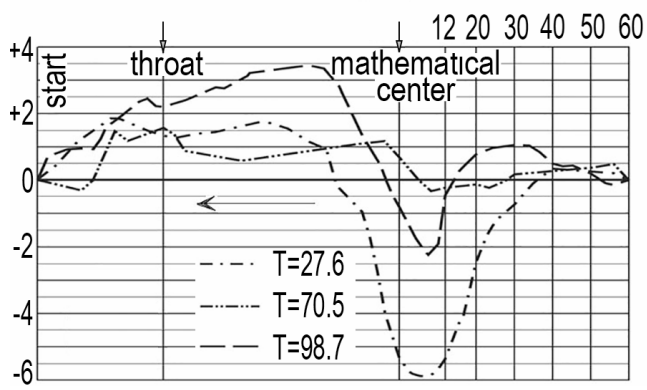

movement in the direction of the branching

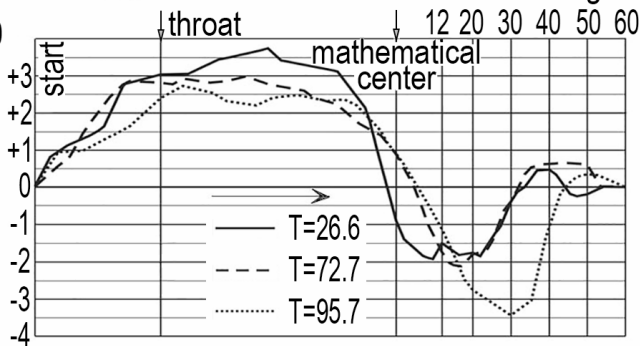

Fig. 3. Average vertical irregularities for the switch frogs P65, mark of crossings $1 / 9$ at different stages of operation.

\section{Estimation of vertical force dynamics on the switch frogs}

Values of dynamic vertical forces acting on the switch frog from wheels of the rolling stock in the zone of irregularities can be determined by the formula:

$$
P_{d y n}=P_{s t}+\Delta P_{k}
$$

The static force $P_{s t}$ is a known value and is taken from references depending on the type of rolling stock. The dynamic addition of vertical force from the influence of irregularity on the rolling surface of the frog sections $\Delta P_{k}$ is as a function from the parameters of irregularities - the depth and slopes of irregularity, and operating conditions - capacity tonnage, speed and the train direction:

$$
\Delta P_{k}=f\left(i_{z}, \Sigma i, h, V\right)
$$

In the formula (2): $i_{z}$ - the opposite slope, $\% ; \Sigma i-$ the total slope on the fracture of irregularities, $\% ;, h$-depth of the irregularity, $\mathrm{mm} ; V$ - speed, $\mathrm{km} / \mathrm{h}$.

Parameters of irregularities $i_{z}, \Sigma i, h$ and conditions of operation of switch frogs: speed of movement $V$, capacity tonnage $T$, prevailing train direction - trailing or facing are the necessary information for calculating dynamic vertical forces.

As a calculation model, the flat longitudinal diagram of the interaction between the track and the 4-axle wagon, proposed in the article $[2,10]$ is presented in Fig. 4. As a disturbing factor in this scheme, irregularities on the surface of frog rolling have been determined. 
The accepted calculation scheme of interaction describes the oscillatory process of the mechanical system of the vehicle-track sufficiently fully and with correctly choosing of design parameters of the track and the vehicle allows to obtain the result with the necessary accuracy, which satisfies the practical goals of the problem.

The peculiarity of the accepted calculation scheme is consideration of the mutual influence on the deflection under each of the considered wheels, the adjoining wheel in the trolley through the general support for wheels - the switch frog.

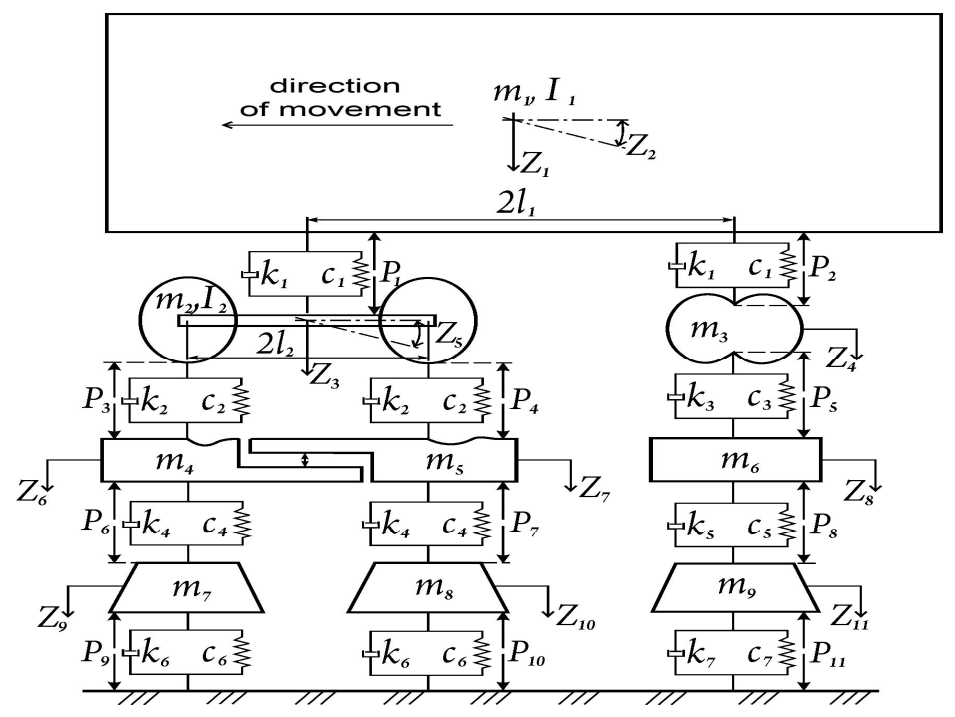

Fig. 4. The scheme of interaction of the switch frog and vehicle in the long vertical surface.

Differential equations of the oscillation process according to the described mechanical system «vehicle-track» on the surface of the rail thread (Fig. 4) due to d'Alembert's principle can be represented as a system of 11 equations (3) [2, 4]. The forces of vertical interaction $P_{i}$, included in this system of differential equations, are determined from dependencies (4).

$$
\left.\begin{array}{l}
m_{1} Z_{1}^{\prime \prime}+P_{1}+P_{2}=0 \\
I_{1} Z_{2}^{\prime \prime}+P_{2} l_{1}+P_{1} l_{1}=0 \\
\left(m_{21}+m_{22}\right) Z_{3}^{\prime \prime}-P_{1}+P_{3}+P_{4}=0 \\
m_{3} Z_{4}^{\prime \prime}-P_{2}+P_{5}=0 \\
I_{2} Z_{5}^{\prime \prime}-P_{3} l_{2}+P_{4} l_{2}=0 \\
m_{4} Z_{6}^{\prime \prime}-P_{3}+P_{6}+\delta_{6-7} P_{7}=0 \\
m_{5} Z_{7}^{\prime \prime}-P_{4}+P_{7}+\delta_{7-6} P_{6}=0 \\
m_{6} Z_{8}^{\prime \prime}-P_{5}+P_{8}=0 \\
m_{7} Z_{9}^{\prime \prime}-P_{6}-\delta_{6-7} P_{7}+P_{9}=0 \\
m_{8} Z_{10}^{\prime \prime}-P_{7}-\delta_{7-6} P_{6}+P_{10}=0 \\
m_{9} Z_{11}^{\prime \prime}-P_{8}+P_{11}=0
\end{array}\right\}(3) \quad \begin{aligned}
& P_{1}=c_{1}\left(Z_{1}-Z_{3}-l_{1} Z_{2}\right)+k_{1}\left(Z_{1}^{\prime}-Z_{3}^{\prime}-l_{1} Z_{2}^{\prime}\right) \\
& P_{2}=c_{1}\left(Z_{1}-Z_{4}-l_{1} Z_{2}\right)+k_{1}\left(Z_{1}^{\prime}-Z_{4}^{\prime}-l_{2} Z_{2}^{\prime}\right) \\
& P_{3}=c_{2}\left(Z_{3}-Z_{6}-l_{2} Z_{5}-\eta_{1}\left(V_{t}\right)\right)+k_{2}\left(Z_{3}^{\prime}-Z_{4}^{\prime}-l_{2} Z_{5}^{\prime}-\eta_{1}\left(V_{t}\right)\right) \\
& P_{4}=c_{2}\left(Z_{3}-Z_{7}+l_{2} Z_{5}-\eta_{2}\left(V_{t}\right)\right)+k_{2}\left(Z_{3}^{\prime}-Z_{7}^{\prime}-l_{2} Z_{5}^{\prime}-\eta_{2}\left(V_{t}\right)\right) \\
& P_{5}=c_{3}\left(Z_{4}-Z_{8}-\eta_{3}\left(V_{t}\right)\right)+k_{3}\left(Z_{3}^{\prime}-Z_{8}^{\prime}-\eta_{3}\left(V_{t}\right)\right) \\
& P_{6}=c_{4}\left(Z_{6}-Z_{9}\right)+k_{4}\left(Z_{6}^{\prime}-Z_{9}^{\prime}\right) \\
& P_{7}=c_{4}\left(Z_{7}-Z_{10}\right)+k_{4}\left(Z_{7}^{\prime}-Z_{10}^{\prime}\right) \\
& P_{8}=c_{5}\left(Z_{8}-Z_{11}\right)+k_{5}\left(Z_{8}^{\prime}-Z_{11}^{\prime}\right) \\
& P_{9}=c_{6} Z_{9}+k_{6} Z_{9}^{\prime} \\
& P_{10}=c_{6} Z_{10}+k_{6} Z_{10}^{\prime} \\
& P_{11}=c_{6} Z_{11}+k_{7} Z_{11}^{\prime}
\end{aligned}
$$

In the scheme (Fig. 4) and equations ( 3 and 4 ) there are following notations: $m$ - mass of system elements; $Z_{i}$ - vertical movements of masses, their first and second derivatives; $c_{i}$ and $k_{i}$-corresponding deflection rate of elastic bonds and coefficients of viscous friction of dissipative viscous elements between the mechanical system; $\delta_{6-7}=\delta_{7-6}$ - ordinates of the influence line on the displacement under the force $P_{6}$ on the strength of $P_{7}$ and vice versa; $\eta$ 
$(V t)$ - functions of irregularities under the corresponding wheels of the vehicle; $I$ moments of inertia and trolley; $2 l_{l}$ - distance between points of the body restraint on the trolley; $2 l_{2}$ - the distance between axles of wheels in the trolley.

The practical implementation of this mathematical model can be performed in any software environment that allows solving mathematical and engineering problems.

Before the forces calculating, the basic elastic-dynamic parameters of the track and the rolling stock for the specific operating conditions have been determined, the speed of motion, initial conditions of the oscillation process and characteristics of irregularities were determined.

The solution of the equations systems (3), (4) is as a function of the distribution of all these interaction forces on the length of irregularities on the switch frog. The practical interest is in the interaction forces at the level of contact points "wheel-frog" $P$, which can be taken as an assessment criterion for the safe operation of the structure. Analysis of the calculation results showed that the largest values of dynamic forces arise under the first wheel of the calculation trolley.

One of the results obtained in calculating dynamic deflections and vertical dynamic forces $P$ between the 1 st wheel and the frog in rolling of the vertical irregularity with speed $V=40,60,80 \mathrm{~km} / \mathrm{h}$, for switch frogs P50, mark of crossings $1 / 9$ with average deterioration (deterioration $\approx 3.0 \mathrm{~mm}$ ) and trailing direction of movement is presented in Fig. 5 .
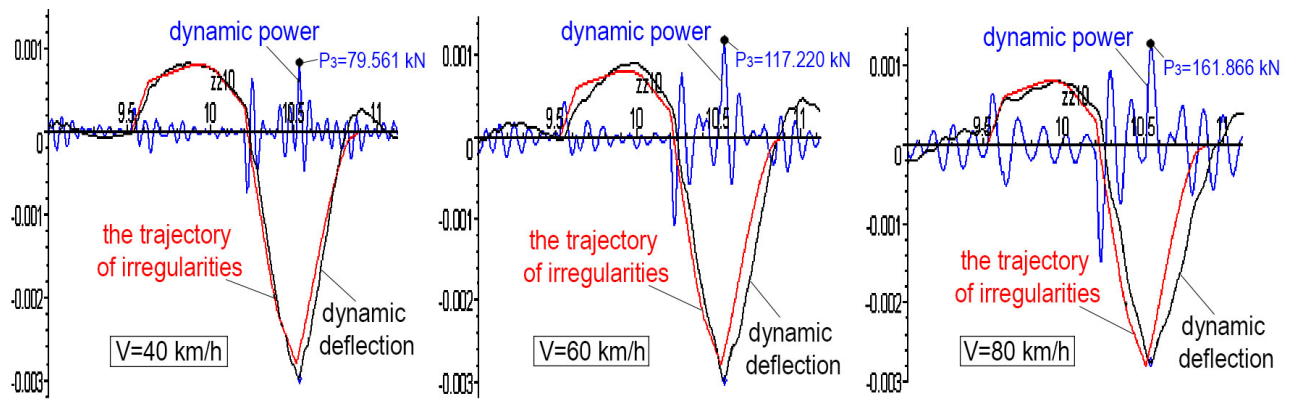

Fig. 5. Distribution of vertical dynamic forces and dynamic deflections of the rail thread in the contact of the wheel and rails on the switch frogs.

For practical implementation of results and the use of force dynamics on the switches frogs in predicting $[2,10]$ there had been proposed the algorithm for establishing functions of the connection for forces of interaction with the parameters of irregularities on the witch frogs at different stages of operation (2).

1. Finding equations of correlation connection $P=f\left(i_{z}\right), P=f(\Sigma i)$ i $P=f\left(h_{\max }\right)$.

2. On the basis of the relation equations $P=f\left(i_{z}\right), P=f(\Sigma i)$ i $P=f\left(h_{\max }\right)$ constructing the functional dependences $P=f(V)$ (differentiated for $i_{z}$ and $\Sigma i$ ) and formulating the equations of relation $P=f(V)$.

3. The cooperative solution of relation equations $P=f\left(i_{z}\right), P=f(\Sigma i)$ i $P=f(V)$ for the case of the analysis of the counter $i_{z}$ and the total slopes of the $\Sigma i$ irregularities. Combination the bifunctional equations $P=f\left(i_{z}, V\right), P=f(\Sigma i, V)$ differentiated by $i_{z}, \Sigma i$ and $V$.

4. The graph-analytic analysis of equations $P=f\left(i_{z}, V\right), P=f(\Sigma i, V)$. Definition of functional dependences of coefficients with arguments and free members of equations from arguments.

5. Solving equations of functional dependences of the universal type (2) separately for switch frogs with different operating conditions.

After the algorithm realization, there have been obtained ultimate universal equations of the form (2) establishing the functional connection of the dynamic interaction forces $P$ with 
the parameters of the vertical irregularity $\left(i_{z}, \Sigma i, h\right)$ and the speed of the vehicle $V$ for the switch frogs.

In the final view, the practical formula for determining the dynamic forces of switch frogs P50, mark of crossings $1 / 9$ due to the medium deterioration condition is:

$$
P=k_{1} \Sigma i+k_{2} \Sigma i V+k_{3} \Sigma i V^{2}+k_{4} V^{2}+k_{5} V+k_{6} i_{z}+k_{7} i_{z} V+k_{8} i_{z} V^{2}+k_{9} h+k_{10} h V+k_{11} h V^{2}+k_{12}
$$

The comparative analysis of results in calculating the forces obtained by mathematical modeling and equations (3) shows a rather close correlation. The maximum deviations in the calculation results are $5-15 \%$.

\section{Conclusions}

The comparative analysis of the average vertical trajectories of rolling on the switch frogs P65 and P50, mark of crossings 1/9 on wooden beams, laid down in the main tracks of the underground railway, allowed to establish main differences, trends, regularities of the development in the track operation and set up the following conclusions.

The shape and parameters of irregularities for the switch frogs P50 and P65 are not significantly different.

The basic form of the irregularity is formed during the initial period of exploitation after the 30-35 million tons capacity, the form is being changed insignificantly in the future but the depth, slope and length of the irregularity are changed.

The qualitative influence on the form and location of the deepest place on the switch frog is set by the prevailing direction of the train movement. Therefore, the switch frogs with trailing or facing movement directions should be considered separately.

According to the known form and parameters of vertical irregularities, additional dynamic forces can be determined at different speeds.

The suggested dependences (3) are universal and allow determining the dynamic forces $\mathrm{P}$ due to the complex of parameters of vertical irregularities on the switch frogs (2) and the established train speeds $\mathrm{V}$ for specific structures under given operating conditions. In practice, the use of the obtained equations does not require complex calculations of elasticdynamic characteristics and the system solution of differential equations.

By the values of permissible vertical forces, it is possible to set permissible speeds of trains on the switch frogs and switches in general $[V]=f\left[P_{\max }\right]$. The recommended values of permissible dynamic forces $\left[P_{\max }\right]$ for the switch frogs under durability conditions of structures have been established $[2,10,11]$ and the amount is $\left[P_{\max }\right] \leq 450-490 \mathrm{\kappa H}$. Accordingly, the permissible level of the dynamic addition of vertical forces (1) from the irregularities influence on the rolling surface must be limited to the switch frogs by the meaning $\left[\Delta P_{\max }\right]=370 \div 410(\mathrm{\kappa H})$.

Maximum forces did not exceed the permissible values for all above-mentioned switch frogs.

\section{References}

1. S.V. Amelin, V.I. Abrosimov, N. N. Elsakov, E. K. Smykov, L. N. Frolov, V. F. Yakovlev, Improvement of the conduct of switch economy (Transport, 1983)

2. E. I. Danilenko, Theoretical bases and practical methods of calculation of durability and deterioration of intersections and connections of railways of industrial transport (Kyiv, 1992)

3. E.I. Danilenko, V.D. Boyko, KUETT, 10, 52-60 (2006) 
4. V. Kovalchuk, M. Sysyn, Yu. Hnativ, O. Bal, B. Parneta, A. Pentsak, ETS, 1 (92), 3342 (2018)

5. V. Boyko V. Molchanov T. Artiukhovych, UkrSURT, 169, 39-47 (2017)

6. K. Aniołek, J. Herian, JTE, 139, 533-539, (2013)

7. Z. Zeng, Z. Yu, X. Chen, JVS, 28, 40-45 (2009)

8. J. Drozdziel \& B. Sowinski, CR, VII, 673-682 (2000)

9. Jingmang Xu, Ping Wang, Li Wang, SAGE J, 7 (2016)

10. E.I. Danilenko, S.D. Taranenko, A.P. Kutah, Switches of Ukrainian railways (Transport of Ukraine, 2001)

11. E.I. Danilenko, V.V. Rybkin, Rules for calculating the railway track for durability and stability (Transport of Ukraine, 2005) 